\title{
Influence of thermal models on the YORP effect
}

\author{
Oleksiy Golubov ${ }^{1,2}$ and Yurij N. Krugly ${ }^{2}$ \\ ${ }^{1}$ Astronomisches Rechen-Institut, Zentrum für Astronomie der Universität Heidelberg, \\ Mönchhofstraße 12-14, 69120 Heidelberg, Germany, \\ ${ }^{2}$ Institute of Astronomy of Kharkiv National University, Sumska Str. 35, 61022 Kharkiv, \\ Ukraine, \\ email: golubov@ari.uni-heidelberg.de
}

\begin{abstract}
In 1-D heat conductivity model, the YORP acceleration is proved to be independent of the asteroid's heat properties. Considering small structures on the surface of an asteroid breaks 1-D model. A new force appears, pulling the asteroid's surface parallel to itself. Its effect on the asteroid's rotation is called tangential YORP, or TYORP.
\end{abstract}

Keywords. asteroids: rotation

Normal YORP effect (or NYORP) is produced by recoil forces normal to the surface of an asteroid, if the asteroid is asymmetric enough, so that these forces do not compensate each other. This effect was first considered by Rubincam (2000) in the model of zero heat conductivity. Later it was proved that the NYORP acceleration is the same for any 1-dimensional heat conductivity model (Golubov \& Krugly 2010; Bottke et al. 2006)). It means that Rubincam's approximation gives precise results for any heat conductivity, and allows to derive simple analytic expression for the NYORP acceleration (Golubov \& Krugly 2010; Steinberg \& Sari 2011).

In contrast, if small structures on the surface of an asteroid are considered (e. g. decimetre-sized stones), 1-D heat conductivity model is no longer valid, and new behaviour arizes. Even though eastern and western sides of a stone absorb the same amount of energy, they can emit different energies (due to nonlinearity of boundary conditions of the heat conductivity equation). Thus the two sides experiences different recoil forces, adding up to push the stone in the direction tangential to the surface, and to produce the so-called tangential YORP (or TYORP), acting on the surface as a whole.

The tangential force experiencing by each surface element of an asteroid is much smaller than the normal force, but influences of the two forces on the rotation rate are estimated to be comparable (Golubov \& Krugly 2012). This has two reasons: first, NYORP forces have bigger lever arms; second, NYORP torques of different surface elements add up rather than subtract, so that even a perfectly symmetric asteroid can experience TYORP. Therefore, TYORP and NYORP may be equally importand for evolution of asteroids.

\section{References}

Breiter, S., Bartczak, P., \& Czekaj, M. 2010,MNRAS 408, 1576

Golubov O. \& Krugly Yu. 2012, APJL, 752, L11

Golubov A. \& Krugly Yu., 2010. In: A. M. Finkelstein, W. F. Huebner, V. A. Shor (Eds.), Protecting the Earth against collisions with asteroids and comet nuclei. Proceedings of Asteroid-Comet Hazard, St. Petersburg, 21-25 Sept., 2009. Nauka, St. Petersburg, pp. 9094

Rubincam, D. P. 2000, Icarus, 148, 2

Steinberg, E. \& Sari, R. 2011, AJ 141:55 